\title{
Non-oscillation of perturbed half-linear differential equations with sums of periodic coefficients
}

Petr Hasil* and Michal Veselý

\section{"Correspondence:} hasil@mail.muni.cz Department of Mathematics and Statistics, Masaryk University, Kotláŕská 2, Brno, 611 37, Czech Republic

\begin{abstract}
We investigate perturbed second order Euler type half-linear differential equations with periodic coefficients and with the perturbations given by the finite sums of periodic functions which do not need to have any common period. Our main interest is to study the oscillatory properties of the equations in the case when the coefficients give exactly the critical oscillation constant. We prove that any of the considered equations is non-oscillatory in this case.
\end{abstract}

MSC: 34C 10; 34C15

Keywords: half-linear equations; oscillation theory; conditional oscillation; Prüfer angle; Riccati equation

\section{Introduction}

The aim of this paper is to contribute to the rapidly developing theory of conditionally oscillatory half-linear differential equations. Our paper is organized to three sections. In this section, we recall the notion of the so-called conditional oscillation and we give a historical background of the topic. In the second section, the reader can find the considered equations together with the description of the used methods (the Riccati and Prüfer transformations). These methods lead to the equation for the Prüfer angle which is the main tool in our investigation. Finally, in the last section, we state lemmas, results, corollaries, and examples.

Let us begin with the concept of the conditional oscillation for half-linear differential equations. We consider the equation

$$
\left[r(t) \Phi\left(x^{\prime}\right)\right]^{\prime}+\gamma c(t) \Phi(x)=0, \quad \Phi(x)=|x|^{p-1} \operatorname{sgn} x, \quad p>1,
$$

where $\gamma$ is a given real constant, coefficients $r$ and $c$ are continuous functions, and $r$ is positive. We say that (1.1) is conditionally oscillatory if there exists a positive constant $\Gamma$ such that (1.1) is oscillatory for $\gamma>\Gamma$ and non-oscillatory for $\gamma<\Gamma$. Such a constant $\Gamma$ is called the critical (oscillation) constant of (1.1).

Now we collect the milestones in the theory of the conditional oscillation with respect to the topic of our paper. It appears that appropriate half-linear equations for the study of

(c) 2015 Hasil and Veselý. This article is distributed under the terms of the Creative Commons Attribution 4.0 International License (http://creativecommons.org/licenses/by/4.0/), which permits unrestricted use, distribution, and reproduction in any medium, provided you give appropriate credit to the original author(s) and the source, provide a link to the Creative Commons license, and indicate if changes were made. 
the conditional oscillation are the Euler type equations, i.e., the equations written in the form

$$
\left[r(t) \Phi\left(x^{\prime}\right)\right]^{\prime}+\frac{\gamma s(t)}{t^{p}} \Phi(x)=0 .
$$

The conditional oscillation (as well as many other areas in the oscillation theory) of half-linear equations originates from the oscillation theory of linear differential equations. The first result about the conditional oscillation of the considered differential equations was obtained by Kneser in [1], where the oscillation constant $\Gamma=1 / 4$ was found for the equation

$$
x^{\prime \prime}+\frac{\gamma}{t^{2}} x=0 .
$$

More than 100 years later, in [2, 3], the above result concerning (1.2) was extended for the linear equations

$$
\left[r(t) x^{\prime}\right]^{\prime}+\frac{\gamma s(t)}{t^{2}} x=0
$$

with positive $\alpha$-periodic coefficients $r$, $s$, where the critical constant is

$$
\Gamma=\frac{\alpha^{2}}{4}\left(\int_{0}^{\alpha} \frac{\mathrm{d} \tau}{r(\tau)}\right)^{-1}\left(\int_{0}^{\alpha} s(\tau) \mathrm{d} \tau\right)^{-1} .
$$

Next, in [4], it was proved that (1.3) is non-oscillatory in the critical case $\gamma=\Gamma$. For other related results, we refer to [5-7].

In the field of half-linear equations, the basic critical constant

$$
\Gamma=\left(\frac{p-1}{p}\right)^{p}
$$

for the equation

$$
\left[\Phi\left(x^{\prime}\right)\right]^{\prime}+\frac{\gamma}{t^{p}} \Phi(x)=0
$$

comes from [8] (see also [9]). Then, in [10-12], the conditional oscillation was proved for more general equations of the form

$$
\left[r(t) \Phi\left(x^{\prime}\right)\right]^{\prime}+\frac{\gamma s(t)}{t^{p}} \Phi(x)=0 .
$$

Especially, the critical constant of (1.6) with positive $\alpha$-periodic functions $r, s$ was identified as (cf. (1.4), (1.5))

$$
\Gamma_{1}=\left(\frac{\alpha(p-1)}{p}\right)^{p}\left(\int_{0}^{\alpha} r^{\frac{1}{1-p}}(\tau) \mathrm{d} \tau\right)^{1-p}\left(\int_{0}^{\alpha} s(\tau) \mathrm{d} \tau\right)^{-1}
$$

in [10]. For the literature and an overview of the theory concerning half-linear (differential) equations, see $[13,14]$. 
Let us turn our attention to the perturbed Euler type equations. The linear case of such equations with periodic coefficients is studied in $[4,5]$. The half-linear case is treated in [15], where the equations

$$
\left[r(t) \Phi\left(x^{\prime}\right)\right]^{\prime}+\left[\gamma s(t)+\frac{\mu d(t)}{\log ^{2} t}\right] \frac{\Phi(x)}{t^{p}}=0
$$

are analyzed for positive $\alpha$-periodic coefficients $r, s$, and $d$. There it is proved that, in the critical case $\gamma=\Gamma_{1}$ (see (1.7)), (1.8) is oscillatory for

$$
\mu>\Gamma_{2}:=\frac{\alpha^{p}}{2}\left(\frac{p-1}{p}\right)^{p-1}\left(\int_{0}^{\alpha} r^{\frac{1}{1-p}}(\tau) \mathrm{d} \tau\right)^{1-p}\left(\int_{0}^{\alpha} d(\tau) \mathrm{d} \tau\right)^{-1}
$$

and non-oscillatory for $\mu<\Gamma_{2}$. For further generalizations, we refer to [16-19] (see also [20]).

In this paper, we are interested in the case when the perturbation is also in the differential term and both of the perturbations are sums of periodic functions. In contrast with the situation common in the literature, the functions in the perturbations do not need to have any common period and can change sign. We prove that all considered equations are nonoscillatory in the critical case. According to the best of our knowledge, this result is new also in the linear case (i.e., for $p=2$ ).

Concerning the conditional oscillation of Euler type linear and half-linear equations, several results are known in the discrete case as well. We point out at least papers [21-23] for difference equations and $[24,25]$ for dynamic equations on time scales. Note that, in the critical case, any of the discrete (and the time scale) counterparts of the above mentioned results is not known even for equations with periodic coefficients.

\section{Preliminaries}

This section is devoted to the description of the considered equations, the corresponding Riccati equations, and to the modified Prüfer angle which is the main method in our processes. We also mention basic definitions and observations which will be essentially applied later.

Throughout the paper, let $p>1$ be arbitrarily given. We use the standard notation $\mathbb{R}_{a}:=[a, \infty)$ and the symbol $q$ denotes the number conjugated with $p(i . e ., p+q=p q)$. We consider the Euler type half-linear equations expressed as

$$
\left[R^{-\frac{p}{q}}(t) \Phi\left(x^{\prime}\right)\right]^{\prime}+S(t) \frac{\Phi(x)}{t^{p}}=0, \quad \Phi(x)=|x|^{p-1} \operatorname{sgn} x,
$$

where $R, S: \mathbb{R}_{\mathrm{e}} \rightarrow \mathbb{R}$ (e stands for the base of the natural logarithm log) are continuous functions such that $R$ is positive and bounded and $S$ is bounded. Note that the power $-p / q$ in the differential term does not mean any loss of generality (see also [17]).

Our main objective is to give a non-oscillation criterion for the half-linear differential equations in the form

$$
\left[\left(r_{0}(t)+\frac{r_{1}(t)}{\log ^{2} t}\right)^{-\frac{p}{q}} \Phi\left(x^{\prime}\right)\right]^{\prime}+\left(s_{0}(t)+\frac{s_{1}(t)}{\log ^{2} t}\right) \frac{\Phi(x)}{t^{p}}=0,
$$


where $r_{0}, r_{1}, s_{0}, s_{1}: \mathbb{R}_{a} \rightarrow \mathbb{R}, a \geq \mathrm{e}$, are continuous functions such that $r_{0}$ is positive and $\alpha$-periodic, $s_{0}$ is $\alpha$-periodic, and

$$
r_{1}(t)=\sum_{i=1}^{n} R_{i}(t), \quad s_{1}(t)=\sum_{i=1}^{n} S_{i}(t), \quad t \geq a,
$$

for arbitrarily given periodic continuous functions $R_{i}$ and $S_{i}$ with periods $\alpha_{i}$ and $\beta_{i}$, respectively. Of course, we can assume that all considered periods $\alpha, \alpha_{i}, \beta_{i}$ are positive and that some of functions $R_{i}, S_{i}$ are identically zero.

At this place, we recall the definition of mean values for continuous functions as a tool that helps us to identify the critical case for studied equations. Let a continuous function $f: \mathbb{R}_{a} \rightarrow \mathbb{R}$ be such that the limit

$$
M(f):=\lim _{t \rightarrow \infty} \frac{1}{t} \int_{b}^{b+t} f(s) \mathrm{d} s
$$

is finite and exists uniformly with respect to $b \in \mathbb{R}_{a}$. The number $M(f)$ is called the mean value of $f$. It is seen that functions $r_{1}, s_{1}$ given in (2.3) have mean values

$$
M\left(r_{1}\right)=\sum_{i=1}^{n} M\left(R_{i}\right), \quad M\left(s_{1}\right)=\sum_{i=1}^{n} M\left(S_{i}\right) .
$$

Concerning the presented results, we will assume that $M\left(r_{1}\right), M\left(s_{1}\right) \geq 0$.

In fact, we study oscillatory properties of the equation

$$
\left[\left(r_{0}(t)+\frac{\sum_{i=1}^{m} R_{i}(t)}{\log ^{2} t}\right)^{-\frac{p}{q}} \Phi\left(x^{\prime}\right)\right]^{\prime}+\left(s_{0}(t)+\frac{\sum_{i=1}^{n} S_{i}(t)}{\log ^{2} t}\right) \frac{\Phi(x)}{t^{p}}=0
$$

with periodic coefficients $R_{1}, \ldots, R_{m}, S_{1}, \ldots, S_{n}$ on $\mathbb{R}_{a}$ at infinity (i.e., value $a$ is large enough) when $\sum_{i=1}^{m} M\left(R_{i}\right) \geq 0, \sum_{i=1}^{n} M\left(S_{i}\right) \geq 0$. For simplicity, we will consider (2.2) only in the critical case (see the below given Theorem 3.4 and $[12,16-18]$ ) given by

$$
\begin{aligned}
& {\left[M\left(r_{0}\right)\right]^{\frac{p}{q}} M\left(s_{0}\right)=\frac{1}{\alpha^{p}}\left(\int_{a}^{a+\alpha} r_{0}(\tau) \mathrm{d} \tau\right)^{\frac{p}{q}}\left(\int_{a}^{a+\alpha} s_{0}(\tau) \mathrm{d} \tau\right)=q^{-p},} \\
& M\left(s_{1}\right)\left[M\left(r_{0}\right)\right]^{\frac{p}{q}}+\frac{p}{q^{p+1}} M\left(r_{1}\right)\left[M\left(r_{0}\right)\right]^{-1}=\frac{q^{1-p}}{2},
\end{aligned}
$$

i.e.,

$$
\lim _{t \rightarrow \infty}\left[\frac{1}{t^{p}}\left(\int_{a}^{a+t} s_{1}(\tau) \mathrm{d} \tau\right)\left(\int_{a}^{a+t} r_{0}(\tau) \mathrm{d} \tau\right)^{\frac{p}{q}}+\frac{p \int_{a}^{a+t} r_{1}(\tau) \mathrm{d} \tau}{q^{p+1} \int_{a}^{a+t} r_{0}(\tau) \mathrm{d} \tau}\right]=\frac{q^{1-p}}{2} .
$$

Then (see the below given Theorem 3.5), we formulate the general result about the oscillation and non-oscillation of (2.5).

To study (2.2), we will consider the equation

$$
\left[\left(M\left(r_{0}\right)+\frac{M\left(r_{1}\right)}{\log ^{2} t}+\frac{1}{\log ^{4} t}\right)^{-\frac{p}{q}} \Phi\left(x^{\prime}\right)\right]^{\prime}+\left(M\left(s_{0}\right)+\frac{M\left(s_{1}\right)}{\log ^{2} t}+\frac{1}{\log ^{4} t}\right) \frac{\Phi(x)}{t^{p}}=0
$$


with constant coefficients and we will also use the notations

$$
\begin{array}{ll}
r_{0}^{+}:=\max \left\{r_{0}(t) ; t \in \mathbb{R}_{a}\right\}, & r_{1}^{+}:=\sup \left\{\left|r_{1}(t)\right| ; t \in \mathbb{R}_{a}\right\}, \\
s_{0}^{+}:=\max \left\{\left|s_{0}(t)\right| ; t \in \mathbb{R}_{a}\right\}, & s_{1}^{+}:=\sup \left\{\left|s_{1}(t)\right| ; t \in \mathbb{R}_{a}\right\},
\end{array}
$$

and

$$
R_{i}^{+}:=\max \left\{\left|R_{i}(t)\right| ; t \in \mathbb{R}_{a}\right\}, \quad S_{i}^{+}:=\max \left\{\left|S_{i}(t)\right| ; t \in \mathbb{R}_{a}\right\}
$$

for each $i$.

As we have already mentioned above, the main method used in this paper is the modified Prüfer angle. To clarify this method, we need some basic properties of the half-linear trigonometric functions. We denote

$$
\pi_{p}:=\frac{2 \pi}{p \sin \frac{\pi}{p}}
$$

and consider the initial value problem

$$
\left[\Phi\left(x^{\prime}\right)\right]^{\prime}+(p-1) \Phi(x)=0, \quad x(0)=0, \quad x^{\prime}(0)=1 .
$$

The odd $2 \pi_{p}$-periodic extension of the solution of (2.12) is called the half-linear sine function and is usually denoted by $\sin _{p}$. The half-linear cosine function is defined as the derivative of the half-linear sine function and it is denoted by $\cos _{p}$. The needed properties of the half-linear trigonometric functions for our purpose are the validity of the half-linear Pythagorean identity

$$
\left|\sin _{p} y\right|^{p}+\left|\cos _{p} y\right|^{p}=1, \quad y \in \mathbb{R}
$$

and the boundedness of these functions given by (see (2.13))

$$
\left|\cos _{p} y\right|^{p} \leq 1, \quad\left|\Phi\left(\cos _{p} y\right) \sin _{p} y\right| \leq 1, \quad\left|\sin _{p} y\right|^{p} \leq 1, \quad y \in \mathbb{R} .
$$

For other properties, we refer, e.g., to [14], Section 1.1.2.

To introduce the notion of the modified half-linear Prüfer angle, we consider the concept of the Riccati equation. Using the transformation

$$
w(t)=R^{-\frac{p}{q}}(t) \Phi\left(\frac{x^{\prime}(t)}{x(t)}\right)
$$

where $x$ is a non-trivial solution of (2.1), we directly obtain the so-called Riccati equation

$$
w^{\prime}+\frac{S(t)}{t^{p}}+(p-1) R(t)|w|^{q}=0
$$

associated to (2.1).

Hence, we can introduce the modified half-linear Prüfer transformation as follows:

$$
x(t)=\rho(t) \sin _{p} \varphi(t), \quad x^{\prime}(t)=\frac{R(t) \rho(t)}{t} \cos _{p} \varphi(t) .
$$


Our aim is to obtain an equation for the Prüfer angle $\varphi$. Therefore, we sketch its derivation at this place. For more details, see [15]. Let us consider a solution $w$ of (2.15). Using (2.14) and (2.16), we have

$$
v:=t^{p-1} w=\Phi\left(\frac{\cos _{p} \varphi}{\sin _{p} \varphi}\right)
$$

which, together with the fact that $\sin _{p}$ is a solution of the initial value problem in (2.12), leads to

$$
v^{\prime}=(1-p)\left[1+\left|\frac{\cos _{p} \varphi}{\sin _{p} \varphi}\right|^{p}\right] \varphi^{\prime}
$$

Now we derive $v^{\prime}$ using (2.15) and (2.17). We obtain

$$
\begin{aligned}
v^{\prime} & =\left[t^{p-1} w\right]^{\prime}=(p-1) t^{p-2} w+t^{p-1} w^{\prime} \\
& =\frac{p-1}{t}\left[v-\frac{S(t)}{p-1}-R(t)\left|\frac{\cos _{p} \varphi}{\sin _{p} \varphi}\right|^{p}\right] .
\end{aligned}
$$

Finally, using (2.17) and comparing (2.18) and (2.19), we have

$$
(1-p)\left[1+\left|\frac{\cos _{p} \varphi}{\sin _{p} \varphi}\right|^{p}\right] \varphi^{\prime}=\frac{p-1}{t}\left[\Phi\left(\frac{\cos _{p} \varphi}{\sin _{p} \varphi}\right)-\frac{S(t)}{p-1}-R(t)\left|\frac{\cos _{p} \varphi}{\sin _{p} \varphi}\right|^{p}\right]
$$

which gives (consider the Pythagorean identity (2.13)) the required equation

$$
\varphi^{\prime}(t)=\frac{1}{t}\left[R(t)\left|\cos _{p} \varphi(t)\right|^{p}-\Phi\left(\cos _{p} \varphi(t)\right) \sin _{p} \varphi(t)+S(t) \frac{\left|\sin _{p} \varphi(t)\right|^{p}}{p-1}\right]
$$

associated to (2.1).

In particular, the Prüfer angle $\varphi$ associated to (2.2) via (2.16) satisfies the equation

$$
\begin{aligned}
\varphi^{\prime}(t)= & \frac{1}{t}\left[\left(r_{0}(t)+\frac{r_{1}(t)}{\log ^{2} t}\right)\left|\cos _{p} \varphi(t)\right|^{p}\right. \\
& \left.-\Phi\left(\cos _{p} \varphi(t)\right) \sin _{p} \varphi(t)+\left(s_{0}(t)+\frac{s_{1}(t)}{\log ^{2} t}\right) \frac{\left|\sin _{p} \varphi(t)\right|^{p}}{p-1}\right] .
\end{aligned}
$$

The equation for the Prüfer angle $\varphi$ associated to (2.8) is (see again (2.20))

$$
\begin{aligned}
\varphi^{\prime}(t)= & \frac{1}{t}\left[\left(M\left(r_{0}\right)+\frac{M\left(r_{1}\right)}{\log ^{2} t}+\frac{1}{\log ^{4} t}\right)\left|\cos _{p} \varphi(t)\right|^{p}\right. \\
& \left.-\Phi\left(\cos _{p} \varphi(t)\right) \sin _{p} \varphi(t)+\left(M\left(s_{0}\right)+\frac{M\left(s_{1}\right)}{\log ^{2} t}+\frac{1}{\log ^{4} t}\right) \frac{\left|\sin _{p} \varphi(t)\right|^{p}}{p-1}\right] .
\end{aligned}
$$

For any solution $\varphi$ of (2.20) on $\mathbb{R}_{a}$, we define the function $\psi: \mathbb{R}_{a} \rightarrow \mathbb{R}$ by the formula

$$
\psi(t):=\int_{t}^{t+\sqrt{t}} \frac{\varphi(\tau)}{\sqrt{\tau}} \mathrm{d} \tau, \quad t \geq a
$$


This auxiliary function $\psi$ will play an important role in the rest of our paper. Note that (2.21) and (2.22) are special cases of (2.20). Thus, the above function $\psi$ is introduced also for solutions of (2.21) and (2.22).

\section{Results}

Now we complete necessary statements which we will use to prove the main result. We begin with two known lemmas.

Lemma 3.1 If $\varphi$ is a solution of (2.20) on $\mathbb{R}_{a}$, then the function $\psi: \mathbb{R}_{a} \rightarrow \mathbb{R}$ defined by (2.23) satisfies

$$
|\varphi(t+s)-\psi(t)| \leq \frac{C \log t}{\sqrt{t}}, \quad t \geq a, s \in[0, \sqrt{t}]
$$

for some $C>0$.

Proof See [26], Lemma 3.2.

Lemma 3.2 Let $\varphi$ be a solution of (2.20) on $\mathbb{R}_{a}$. Then there exist $A, c>0$ such that the function $\psi: \mathbb{R}_{a} \rightarrow \mathbb{R}$ defined in (2.23) satisfies the inequality

$$
\begin{gathered}
\mid \psi^{\prime}(t)-\frac{1}{t}\left[\frac{\left|\cos _{p} \psi(t)\right|^{p}}{\sqrt{t}} \int_{t}^{t+\sqrt{t}} R(\tau) \mathrm{d} \tau-\Phi\left(\cos _{p} \psi(t)\right) \sin _{p} \psi(t)\right. \\
\left.+\frac{\left|\sin _{p} \psi(t)\right|^{p}}{(p-1) \sqrt{t}} \int_{t}^{t+\sqrt{t}} S(\tau) \mathrm{d} \tau\right] \mid \leq \frac{A}{t^{1+c}}
\end{gathered}
$$

for all $t>a$.

Proof The lemma comes directly from [26], Lemma 3.4.

Next, we will need the following results.

Lemma 3.3 Let $\varphi$ be a solution of (2.22) on $\mathbb{R}_{a}$. Then the function $\psi: \mathbb{R}_{a} \rightarrow \mathbb{R}$ defined in (2.23) satisfies the inequality

$$
\begin{aligned}
\psi^{\prime}(t) \geq & \frac{1}{t}\left[\left(M\left(r_{0}\right)+\frac{M\left(r_{1}\right)}{\log ^{2} t}\right)\left|\cos _{p} \psi(t)\right|^{p}-\Phi\left(\cos _{p} \psi(t)\right) \sin _{p} \psi(t)\right. \\
& \left.+\left(M\left(s_{0}\right)+\frac{M\left(s_{1}\right)}{\log ^{2} t}\right) \frac{\left|\sin _{p} \psi(t)\right|^{p}}{p-1}+\frac{1}{\log ^{5} t}\right]
\end{aligned}
$$

for all sufficiently large $t$.

Proof From Lemma 3.2, we have

$$
\begin{aligned}
\psi^{\prime}(t) \geq & \frac{1}{t}\left[\frac{\left|\cos _{p} \psi(t)\right|^{p}}{\sqrt{t}} \int_{t}^{t+\sqrt{t}}\left(M\left(r_{0}\right)+\frac{M\left(r_{1}\right)}{\log ^{2} \tau}+\frac{1}{\log ^{4} \tau}\right) \mathrm{d} \tau\right. \\
& -\Phi\left(\cos _{p} \psi(t)\right) \sin _{p} \psi(t)
\end{aligned}
$$




$$
\begin{aligned}
& \left.\quad+\frac{\left|\sin _{p} \psi(t)\right|^{p}}{(p-1) \sqrt{t}} \int_{t}^{t+\sqrt{t}}\left(M\left(s_{0}\right)+\frac{M\left(s_{1}\right)}{\log ^{2} \tau}+\frac{1}{\log ^{4} \tau}\right) \mathrm{d} \tau-\frac{A}{t^{c}}\right] \\
& \geq \frac{1}{t}\left[\left|\cos _{p} \psi(t)\right|^{p}\left(M\left(r_{0}\right)+\frac{M\left(r_{1}\right)}{\log ^{2}(t+\sqrt{t})}+\frac{1}{\log ^{4}(t+\sqrt{t})}\right)\right. \\
& \quad-\Phi\left(\cos _{p} \psi(t)\right) \sin _{p} \psi(t) \\
& \left.\quad+\frac{\left|\sin _{p} \psi(t)\right|^{p}}{p-1}\left(M\left(s_{0}\right)+\frac{M\left(s_{1}\right)}{\log ^{2}(t+\sqrt{t})}+\frac{1}{\log ^{4}(t+\sqrt{t})}\right)-\frac{A}{t^{c}}\right]
\end{aligned}
$$

for all $t>a$. Via the mean value theorem, one can directly compute

$$
0 \leq \limsup _{t \rightarrow \infty} \log ^{2} t\left[\log ^{2}(t+\sqrt{t})-\log ^{2} t\right] \leq \lim _{t \rightarrow \infty} \log ^{2} t \frac{2 \log t}{t} \sqrt{t}=0 .
$$

Thus, we have

$$
\begin{aligned}
\left|\frac{M\left(r_{1}\right)}{\log ^{2}(t+\sqrt{t})}-\frac{M\left(r_{1}\right)}{\log ^{2} t}\right| & \leq M\left(r_{1}\right) \frac{\log ^{2}(t+\sqrt{t})-\log ^{2} t}{\log ^{4} t} \leq \frac{1}{\log ^{6} t} \\
\left|\frac{M\left(s_{1}\right)}{\log ^{2}(t+\sqrt{t})}-\frac{M\left(s_{1}\right)}{\log ^{2} t}\right| & \leq M\left(s_{1}\right) \frac{\log ^{2}(t+\sqrt{t})-\log ^{2} t}{\log ^{4} t} \leq \frac{p-1}{\log ^{6} t}
\end{aligned}
$$

for all large $t$. From (2.13), it is seen that

$$
\max \left\{\left|\sin _{p} y\right|^{p},\left|\cos _{p} y\right|^{p}\right\} \geq \frac{1}{2}, \quad y \in \mathbb{R}
$$

Hence, for large $t$, we have

$$
\frac{\left|\cos _{p} y\right|^{p}}{\log ^{4}(t+\sqrt{t})}+\frac{\left|\sin _{p} y\right|^{p}}{(p-1) \log ^{4}(t+\sqrt{t})}>\frac{2}{\log ^{5} t}, \quad y \in \mathbb{R} .
$$

Altogether, using (3.3), (3.4), and (3.5), we obtain

$$
\begin{aligned}
\psi^{\prime}(t) \geq \frac{1}{t} & {\left[\left(M\left(r_{0}\right)+\frac{M\left(r_{1}\right)}{\log ^{2} t}\right)\left|\cos _{p} \psi(t)\right|^{p}-\Phi\left(\cos _{p} \psi(t)\right) \sin _{p} \psi(t)\right.} \\
& \left.+\left(M\left(s_{0}\right)+\frac{M\left(s_{1}\right)}{\log ^{2} t}\right) \frac{\left|\sin _{p} \psi(t)\right|^{p}}{p-1}+\frac{2}{\log ^{5} t}-\frac{2}{\log ^{6} t}-\frac{A}{t^{c}}\right]
\end{aligned}
$$

for large $t$, which gives (3.1).

Lemma 3.4 Let $\varphi$ be a solution of (2.21) on $\mathbb{R}_{a}$. Then there exists $B>0$ such that the function $\psi: \mathbb{R}_{a} \rightarrow \mathbb{R}$ defined by (2.23) satisfies the inequality

$$
\begin{aligned}
\psi^{\prime}(t) \leq & \frac{1}{t}\left[\left(M\left(r_{0}\right)+\frac{M\left(r_{1}\right)}{\log ^{2} t}\right)\left|\cos _{p} \psi(t)\right|^{p}-\Phi\left(\cos _{p} \psi(t)\right) \sin _{p} \psi(t)\right. \\
& \left.+\left(M\left(s_{0}\right)+\frac{M\left(s_{1}\right)}{\log ^{2} t}\right) \frac{\left|\sin _{p} \psi(t)\right|^{p}}{p-1}+\frac{B}{\log ^{6} t}\right]
\end{aligned}
$$

for all sufficiently large $t$. 
Proof From Lemma 3.2, we know that the inequality

$$
\begin{aligned}
\psi^{\prime}(t) \leq & \frac{1}{t}\left[\frac{\left|\cos _{p} \psi(t)\right|^{p}}{\sqrt{t}} \int_{t}^{t+\sqrt{t}}\left(r_{0}(\tau)+\frac{r_{1}(\tau)}{\log ^{2} \tau}\right) \mathrm{d} \tau-\Phi\left(\cos _{p} \psi(t)\right) \sin _{p} \psi(t)\right. \\
& \left.+\frac{\left|\sin _{p} \psi(t)\right|^{p}}{(p-1) \sqrt{t}} \int_{t}^{t+\sqrt{t}}\left(s_{0}(\tau)+\frac{s_{1}(\tau)}{\log ^{2} \tau}\right) \mathrm{d} \tau+\frac{A}{t^{c}}\right]
\end{aligned}
$$

holds for all $t>a$. It means that it suffices to prove

$$
\begin{aligned}
& \left|\frac{1}{\sqrt{t}} \int_{t}^{t+\sqrt{t}} r_{0}(\tau) \mathrm{d} \tau-M\left(r_{0}\right)\right| \leq \frac{A_{0}}{\sqrt{t}}, \quad\left|\frac{1}{\sqrt{t}} \int_{t}^{t+\sqrt{t}} s_{0}(\tau) \mathrm{d} \tau-M\left(s_{0}\right)\right| \leq \frac{B_{0}}{\sqrt{t}}, \\
& \left|\frac{1}{\sqrt{t}} \int_{t}^{t+\sqrt{t}} \frac{r_{1}(\tau)}{\log ^{2} \tau} \mathrm{d} \tau-\frac{M\left(r_{1}\right)}{\log ^{2} t}\right| \leq \frac{A_{1}}{\log ^{6} t},
\end{aligned}
$$

and

$$
\left|\frac{1}{\sqrt{t}} \int_{t}^{t+\sqrt{t}} \frac{s_{1}(\tau)}{\log ^{2} \tau} \mathrm{d} \tau-\frac{M\left(s_{1}\right)}{\log ^{2} t}\right| \leq \frac{B_{1}}{\log ^{6} t}
$$

for some $A_{0}, B_{0}, A_{1}, B_{1}>0$, and for all large $t$.

Let $f: \mathbb{R} \rightarrow \mathbb{R}$ be an arbitrary continuous periodic function with period $\delta>0$. Let a given number $t$ be sufficiently large and $l \in \mathbb{N}$ be such that $\sqrt{t} \in[l \delta,(l+1) \delta)$. We have

$$
\begin{aligned}
& \left|\frac{1}{\sqrt{t}} \int_{t}^{t+\sqrt{t}} f(\tau) \mathrm{d} \tau-M(f)\right| \\
& \quad \leq\left|\frac{1}{\sqrt{t}} \int_{t}^{t+\sqrt{t}} f(\tau) \mathrm{d} \tau-\frac{1}{\sqrt{t}} \int_{t}^{t+l \delta} f(\tau) \mathrm{d} \tau\right|+\left|\frac{1}{\sqrt{t}} \int_{t}^{t+l \delta} f(\tau) \mathrm{d} \tau-M(f)\right| \\
& \quad \leq\left|\frac{1}{\sqrt{t}} \int_{t+l \delta}^{t+\sqrt{t}} f(\tau) \mathrm{d} \tau\right|+\left|\frac{1}{\sqrt{t}} \int_{t}^{t+l \delta} f(\tau) \mathrm{d} \tau-\frac{1}{l \delta} \int_{t}^{t+l \delta} f(\tau) \mathrm{d} \tau\right| \\
& \quad \leq \frac{\delta \max _{t \in[0, \delta)}|f(t)|}{\sqrt{t}}+\left(\frac{1}{l \delta}-\frac{1}{\sqrt{t}}\right) l \delta M(f) \leq \frac{\delta \max _{t \in[0, \delta)}|f(t)|+\delta M(f)}{\sqrt{t}}
\end{aligned}
$$

Thus, (3.6) is valid for (see (2.9))

$$
A_{0}=\alpha\left[r_{0}^{+}+M\left(r_{0}\right)\right], \quad B_{0}=\alpha\left[s_{0}^{+}+M\left(s_{0}\right)\right]
$$

Since (3.9) is true for any periodic continuous function $f$, we obtain (see (2.3), (2.4), (2.10))

$$
\begin{aligned}
& \left|\frac{1}{\sqrt{t}} \int_{t}^{t+\sqrt{t}} r_{1}(\tau) \mathrm{d} \tau-M\left(r_{1}\right)\right| \\
& \quad=\left|\frac{1}{\sqrt{t}} \int_{t}^{t+\sqrt{t}} \sum_{i=1}^{n} R_{i}(\tau) \mathrm{d} \tau-M\left(\sum_{i=1}^{n} R_{i}\right)\right| \\
& \quad=\left|\sum_{i=1}^{n}\left(\frac{1}{\sqrt{t}} \int_{t}^{t+\sqrt{t}} R_{i}(\tau) \mathrm{d} \tau-M\left(R_{i}\right)\right)\right|
\end{aligned}
$$




$$
\begin{aligned}
& \leq \sum_{i=1}^{n}\left|\frac{1}{\sqrt{t}} \int_{t}^{t+\sqrt{t}} R_{i}(\tau) \mathrm{d} \tau-M\left(R_{i}\right)\right| \\
& \leq \sum_{i=1}^{n} \frac{\alpha_{i} R_{i}^{+}+\alpha_{i} M\left(R_{i}\right)}{\sqrt{t}} .
\end{aligned}
$$

Analogously,

$$
\left|\frac{1}{\sqrt{t}} \int_{t}^{t+\sqrt{t}} s_{1}(\tau) \mathrm{d} \tau-M\left(s_{1}\right)\right| \leq \sum_{i=1}^{n} \frac{\beta_{i} S_{i}^{+}+\beta_{i} M\left(S_{i}\right)}{\sqrt{t}} .
$$

Using (3.10), we have

$$
\begin{aligned}
& \left|\frac{1}{\sqrt{t}} \int_{t}^{t+\sqrt{t}} \frac{r_{1}(\tau)}{\log ^{2} \tau} \mathrm{d} \tau-\frac{M\left(r_{1}\right)}{\log ^{2} t}\right| \\
& \quad \leq\left|\frac{1}{\sqrt{t}} \int_{t}^{t+\sqrt{t}} \frac{r_{1}(\tau)}{\log ^{2} \tau} \mathrm{d} \tau-\frac{1}{\sqrt{t}} \int_{t}^{t+\sqrt{t}} \frac{r_{1}(\tau)}{\log ^{2} t} \mathrm{~d} \tau\right|+\left|\frac{1}{\sqrt{t}} \int_{t}^{t+\sqrt{t}} \frac{r_{1}(\tau)}{\log ^{2} t} \mathrm{~d} \tau-\frac{M\left(r_{1}\right)}{\log ^{2} t}\right| \\
& \quad \leq \frac{r_{1}^{+}}{\sqrt{t}} \int_{t}^{t+\sqrt{t}}\left|\frac{1}{\log ^{2} \tau}-\frac{1}{\log ^{2} t}\right| \mathrm{d} \tau+\frac{1}{\log ^{2} t}\left|\frac{1}{\sqrt{t}} \int_{t}^{t+\sqrt{t}} r_{1}(\tau) \mathrm{d} \tau-M\left(r_{1}\right)\right| \\
& \quad \leq r_{1}^{+}\left[\frac{\log ^{2}(t+\sqrt{t})-\log ^{2} t}{\log ^{4} t}\right]+\frac{1}{\log ^{2} t} \sum_{i=1}^{n} \frac{\alpha_{i} R_{i}^{+}+\alpha_{i} M\left(R_{i}\right)}{\sqrt{t}}
\end{aligned}
$$

for large $t$. Considering (3.2), we obtain (3.7) from (3.12). Analogously, one can obtain (3.8) applying (3.11). Hence, the proof is complete.

Lemma 3.5 Equation (2.8) is non-oscillatory.

Proof The non-oscillation of (2.8) follows from [16], Theorem 4.1 (see also [17]) and the Sturmian half-linear comparison theorem (see, e.g., [14], Theorem 1.2.4). More precisely, from [16], Theorem 4.1 it follows that the equation

$$
\begin{aligned}
& {\left[\left(M\left(r_{0}\right)+\frac{M\left(r_{1}\right)}{\log ^{2} t}+\frac{\varepsilon}{[\log t \cdot \log (\log t)]^{2}}\right)^{-\frac{p}{q}} \Phi\left(x^{\prime}\right)\right]^{\prime}} \\
& \quad+\left(M\left(s_{0}\right)+\frac{M\left(s_{1}\right)}{\log ^{2} t}+\frac{\varepsilon}{[\log t \cdot \log (\log t)]^{2}}\right) \frac{\Phi(x)}{t^{p}}=0
\end{aligned}
$$

is non-oscillatory for any sufficiently small $\varepsilon>0$ (it is described in [17]) and (3.13) is a non-oscillatory majorant of (2.8).

Lemma 3.6 For a solution $\varphi$ of (2.22) on $\mathbb{R}_{a}$, we have

$$
\limsup _{t \rightarrow \infty} \varphi(t)=\limsup _{t \rightarrow \infty} \psi(t)<\infty,
$$

where $\psi$ is introduced in (2.23).

Proof Lemma 3.5 says that any considered solution $\varphi$ is bounded from above. Indeed, it suffices to consider (2.16) and (2.22) when $\sin _{p} \varphi(t)=0$. For details, we can refer, e.g., to [14], Section 1.1.3, $[4,15,19]$. Finally, the equality in (3.14) follows from Lemma 3.1. 
Now we can prove the announced result.

Theorem 3.1 Equation (2.2) with (2.6) and (2.7) is non-oscillatory.

Proof We recall that the non-oscillation of (2.2) is equivalent to the boundedness of a solution $\varphi$ of (2.21) on $\mathbb{R}_{a}$ (see again each one of papers [4, 15] or [19]). In addition, a solution $\varphi$ of (2.21) on $\mathbb{R}_{a}$ is bounded if and only if $\lim \sup _{t \rightarrow \infty} \varphi(t)<\infty$. It is seen from the right-hand side of (2.21) when $\sin _{p} \varphi(t)=0$.

Let sufficiently large $T>a$ be given. Let us consider an arbitrary solution $\varphi$ of (2.21) on $\mathbb{R}_{T}$ and the corresponding function $\psi: \mathbb{R}_{T} \rightarrow \mathbb{R}$ given by (2.23). Lemma 3.4 ensures

$$
\begin{aligned}
\psi^{\prime}(t) \leq & \frac{1}{t}\left[\left(M\left(r_{0}\right)+\frac{M\left(r_{1}\right)}{\log ^{2} t}\right)\left|\cos _{p} \psi(t)\right|^{p}-\Phi\left(\cos _{p} \psi(t)\right) \sin _{p} \psi(t)\right. \\
& \left.+\left(M\left(s_{0}\right)+\frac{M\left(s_{1}\right)}{\log ^{2} t}\right) \frac{\left|\sin _{p} \psi(t)\right|^{p}}{p-1}+\frac{B}{\log ^{6} t}\right], \quad t>T .
\end{aligned}
$$

Thus, we have

$$
\begin{aligned}
\psi^{\prime}(t)< & \frac{1}{t}\left[\left(M\left(r_{0}\right)+\frac{M\left(r_{1}\right)}{\log ^{2} t}\right)\left|\cos _{p} \psi(t)\right|^{p}-\Phi\left(\cos _{p} \psi(t)\right) \sin _{p} \psi(t)\right. \\
& \left.+\left(M\left(s_{0}\right)+\frac{M\left(s_{1}\right)}{\log ^{2} t}\right) \frac{\left|\sin _{p} \psi(t)\right|^{p}}{p-1}+\frac{1}{\log ^{5} t}\right], \quad t>T,
\end{aligned}
$$

because $T$ can be chosen arbitrarily.

We consider the solution $\tilde{\varphi}$ of (2.22) given by the initial condition (see (2.11))

$$
\tilde{\varphi}(T)=\max \{\varphi(T+\sqrt{t}) ; t \in[0, T]\}+\pi_{p}
$$

and the corresponding function $\tilde{\psi}$ given by (2.23). Considering the form of (2.22) and (3.16), one can show that

$$
\psi(T)<\tilde{\psi}(T)
$$

Lemma 3.6 says that (3.14) is valid for $\tilde{\varphi}$ and $\tilde{\psi}$, i.e., we have

$$
\limsup _{t \rightarrow \infty} \tilde{\varphi}(t)=\limsup _{t \rightarrow \infty} \tilde{\psi}(t)<\infty
$$

Lemma 3.3 gives

$$
\begin{aligned}
\tilde{\psi}^{\prime}(t) \geq & \frac{1}{t}\left[\left(M\left(r_{0}\right)+\frac{M\left(r_{1}\right)}{\log ^{2} t}\right)\left|\cos _{p} \tilde{\psi}(t)\right|^{p}-\Phi\left(\cos _{p} \tilde{\psi}(t)\right) \sin _{p} \tilde{\psi}(t)\right. \\
& \left.+\left(M\left(s_{0}\right)+\frac{M\left(s_{1}\right)}{\log ^{2} t}\right) \frac{\left|\sin _{p} \tilde{\psi}(t)\right|^{p}}{p-1}+\frac{1}{\log ^{5} t}\right], \quad t>T .
\end{aligned}
$$

Considering (3.15), (3.17), (3.18), and (3.19), we obtain

$$
\limsup _{t \rightarrow \infty} \psi(t) \leq \limsup _{t \rightarrow \infty} \tilde{\psi}(t)<\infty
$$


Indeed, it suffices to consider the case when $\psi\left(t_{0}\right)=\tilde{\psi}\left(t_{0}\right)$ for any $t_{0}>T$. Using Lemma 3.1, we know that $\varphi$ is bounded from above which implies the non-oscillation of (2.2).

To illustrate our results, we mention examples. We remark that all given examples are not generally solvable using any previously known non-oscillation criteria.

Example 1 Immediately, Theorem 3.1 gives the non-oscillation of several equations. For example, the equations

$$
\begin{aligned}
& {\left[\left(1+\frac{\sin t}{p}+\frac{q^{2}+\sin (\sqrt{2} t)}{2 p \log ^{2} t}\right)^{-\frac{p}{q}} \Phi\left(x^{\prime}\right)\right]^{\prime}+\left(q^{-p}+\sin (5 t)\right) \frac{\Phi(x)}{t^{p}}=0,} \\
& {\left[\left(1+\arctan \left(\sin _{3} t\right)\right)^{-\frac{p}{q}} \Phi\left(x^{\prime}\right)\right]^{\prime}+q^{-p}\left(1+\frac{\pi q|\sin t|}{4 \log ^{2} t}\right) \frac{\Phi(x)}{t^{p}}=0}
\end{aligned}
$$

are non-oscillatory.

Theorem 3.1 implies new results in many special cases. We obtain a new result even for linear equations with constant and periodic coefficients which is formulated as the corollary below.

Corollary 3.1 Let $f, g$ be periodic and continuous functions such that $M(f), M(g) \geq 0$ and $M(f)+M(g)=1$. The equation

$$
\left[\left(1+\frac{f(t)}{\log ^{2} t}\right)^{-1} x^{\prime}\right]^{\prime}+\frac{1}{4 t^{2}}\left(1+\frac{g(t)}{\log ^{2} t}\right) x=0
$$

is non-oscillatory.

Example 2 Let $a \in(0,1)$ and $\varrho, \sigma>1$ be arbitrary. For the linear equations

$$
\begin{aligned}
& {\left[\frac{x^{\prime}}{1+\left(a+\sin _{\varrho} t\right) / \log ^{2} t}\right]^{\prime}+\frac{1+\left(1-a+\sin _{\sigma} t\right) / \log ^{2} t}{4 t^{2}} x=0,} \\
& {\left[\frac{x^{\prime}}{1+\left(a+\sin _{\varrho} t\right) / \log ^{2} t}\right]^{\prime}+\frac{1+\left(1-a+\cos _{\sigma} t\right) / \log ^{2} t}{4 t^{2}} x=0,} \\
& {\left[\frac{x^{\prime}}{1+\left(a+\cos _{\varrho} t\right) / \log ^{2} t}\right]^{\prime}+\frac{1+\left(1-a+\sin _{\sigma} t\right) / \log ^{2} t}{4 t^{2}} x=0,} \\
& {\left[\frac{x^{\prime}}{1+\left(a+\cos _{\varrho} t\right) / \log ^{2} t}\right]^{\prime}+\frac{1+\left(1-a+\cos _{\sigma} t\right) / \log ^{2} t}{4 t^{2}} x=0,}
\end{aligned}
$$

we can apply Corollary 3.1. Thus, the above equations are non-oscillatory.

Now we mention two relevant results.

Theorem 3.2 Letc $: \mathbb{R}_{a} \rightarrow \mathbb{R}$ be a continuous function, for which mean value $M\left(c^{1-q}\right)$ exists and for which

$$
0<\inf _{t \in \mathbb{R}_{a}} c(t) \leq \sup _{t \in \mathbb{R}_{a}} c(t)<\infty
$$


and let $d: \mathbb{R}_{a} \rightarrow \mathbb{R}$ be a continuous function having mean value $M(d)$. Let us consider the equation

$$
\left[c(t) \Phi\left(x^{\prime}\right)\right]^{\prime}+\frac{d(t)}{t^{p}} \Phi(x)=0
$$

and denote

$$
\Lambda:=q^{-p}\left[M\left(c^{1-q}\right)\right]^{1-p}
$$

The following statements hold.

(i) Equation (3.21) is oscillatory if $M(d)>\Lambda$.

(ii) Equation (3.21) is non-oscillatory if $M(d)<\Lambda$.

Proof See [12], Theorem 9.

Theorem 3.3 Let $c_{1}$ be a positive $\alpha$-periodic continuous function, let $d_{1}$ be an $\alpha$-periodic continuous function, and let $c_{2}, d_{2}: \mathbb{R}_{a} \rightarrow \mathbb{R}$ be arbitrary continuous functions for which mean values $M\left(c_{2}\right), M\left(\left|c_{2}\right|\right), M\left(d_{2}\right), M\left(\left|d_{2}\right|\right)$ exist. Let us consider the equation

$$
\left[\left(c_{1}(t)+\frac{c_{2}(t)}{\log ^{2} t}\right)^{-\frac{p}{q}} \Phi\left(x^{\prime}\right)\right]^{\prime}+\left(d_{1}(t)+\frac{d_{2}(t)}{\log ^{2} t}\right) \frac{\Phi(x)}{t^{p}}=0
$$

and denote

$$
\Gamma:=2 q^{p-1} M\left(d_{2}\right)\left[M\left(c_{1}\right)\right]^{\frac{p}{q}}+2 q^{-2} p M\left(c_{2}\right)\left[M\left(c_{1}\right)\right]^{-1} .
$$

Let

$$
c_{1}(t)+\frac{c_{2}(t)}{\log ^{2} t}>0, \quad t \geq a, \quad q^{p} M\left(d_{1}\right)\left[M\left(c_{1}\right)\right]^{\frac{p}{q}}=1 .
$$

The following statements hold.

(i) Equation (3.22) is oscillatory if $\Gamma>1$.

(ii) Equation (3.22) is non-oscillatory if $\Gamma<1$.

Proof See [18], Theorem 5.1, where it suffices to put $n=1$.

Combining Theorems 3.2 and 3.3, we obtain the following one.

Theorem 3.4 The following statements hold.

(i) If $\left[M\left(r_{0}\right)\right]^{\frac{p}{q}} M\left(s_{0}\right)>q^{-p}$, then (2.2) is oscillatory.

(ii) If $\left[M\left(r_{0}\right)\right]^{\frac{p}{q}} M\left(s_{0}\right)<q^{-p}$, then (2.2) is non-oscillatory.

(iii) If $\left[M\left(r_{0}\right)\right]^{\frac{p}{q}} M\left(s_{0}\right)=q^{-p}$ and

$$
M\left(s_{1}\right)\left[M\left(r_{0}\right)\right]^{\frac{p}{q}}+\frac{p}{q^{p+1}} M\left(r_{1}\right)\left[M\left(r_{0}\right)\right]^{-1}>\frac{q^{1-p}}{2},
$$

then (2.2) is oscillatory. 
(iv) If $\left[M\left(r_{0}\right)\right]^{\frac{p}{q}} M\left(s_{0}\right)=q^{-p}$ and

$$
M\left(s_{1}\right)\left[M\left(r_{0}\right)\right]^{\frac{p}{q}}+\frac{p}{q^{p+1}} M\left(r_{1}\right)\left[M\left(r_{0}\right)\right]^{-1}<\frac{q^{1-p}}{2}
$$

then (2.2) is non-oscillatory.

Proof The theorem follows immediately from Theorem 3.2 (parts (i), (ii)) and Theorem 3.3 (parts (iii), (iv)). It suffices to consider the identities $p-1=p / q,(1-q)(-p / q)=1$.

Applying Theorem 3.4, we can improve Theorem 3.1 and Corollary 3.1 into the following more convenient forms. We give illustrating examples as well.

Theorem 3.5 Equation (2.5) is non-oscillatory if and only if

$$
\begin{aligned}
& \lim _{t \rightarrow \infty}\left[\frac{t}{\alpha^{p}}\left(\int_{a}^{a+\alpha} r_{0}(\tau) \mathrm{d} \tau\right)^{\frac{p}{q}}\left(\int_{a}^{a+\alpha} s_{0}(\tau) \mathrm{d} \tau\right)-q^{-p} t\right. \\
& \left.\quad+\frac{1}{t^{p}}\left(\int_{a}^{a+t} \sum_{i=1}^{n} S_{i}(\tau) \mathrm{d} \tau\right)\left(\int_{a}^{a+t} r_{0}(\tau) \mathrm{d} \tau\right)^{\frac{p}{q}}+\frac{p \int_{a}^{a+t} \sum_{i=1}^{m} R_{i}(\tau) \mathrm{d} \tau}{q^{p+1} \int_{a}^{a+t} r_{0}(\tau) \mathrm{d} \tau}\right] \leq \frac{q^{1-p}}{2} .
\end{aligned}
$$

Proof It suffices to consider Theorems 3.1 and 3.4.

Example 3 Let $a, b, c, d>0, a_{1}, a_{2}, a_{3}, b_{1} \neq 0, p=3 / 2$. Let us consider the half-linear equation

$$
\begin{aligned}
& {\left[\frac{1}{\sqrt{a+c\left(\cos \left(a_{1} t\right) \sin \left(a_{1} t\right)+\cos \left(a_{2} t\right)+\sin \left(a_{3} t\right)\right) / \log ^{2} t}} \cdot \frac{x^{\prime}}{\sqrt{\left|x^{\prime}\right|}}\right]^{\prime}} \\
& \quad+\left(b+d\left[\frac{\cos \left(b_{1} t\right) \sin \left(b_{1} t\right)}{\log t}\right]^{2}\right) \frac{x}{\sqrt{t^{3}|x|}}=0 .
\end{aligned}
$$

Theorem 3.5 guarantees the oscillation of (3.23) if $a b^{2}>1 / 27$; and its non-oscillation if $a b^{2}<1 / 27$. We put $a b^{2}=1 / 27$. Since

$$
M(\cos (\alpha t) \sin (\alpha t))=M(\cos (\alpha t))=M(\sin (\alpha t))=0, \quad \alpha \neq 0,
$$

and

$$
M\left([\cos (\alpha t) \sin (\alpha t)]^{2}\right)=\frac{1}{8}, \quad \alpha \neq 0,
$$

we obtain the oscillation of (3.23) for $a d^{2}>16 / 3$ and the non-oscillation in the opposite case $a d^{2} \leq 16 / 3$.

Corollary 3.2 Let $f, g$ be periodic and continuous functions such that $M(f), M(g) \geq 0$. Equation (3.20) is oscillatory if and only if $M(f)+M(g)>1$. 
Example 4 Using Corollary 3.2 (and Theorem 3.5), we can generalize Example 2. For any $a_{1}, a_{2}, b_{1}, b_{2}>0$, and $\varrho, \sigma>1$, the linear equations

$$
\begin{aligned}
& {\left[\frac{x^{\prime}}{a_{1}+\left(b_{1}+\sin _{\varrho} t\right) / \log ^{2} t}\right]^{\prime}+\frac{a_{2}+\left(b_{2}+\sin _{\sigma} t\right) / \log ^{2} t}{4 t^{2}} x=0,} \\
& {\left[\frac{x^{\prime}}{a_{1}+\left(b_{1}+\sin _{\varrho} t\right) / \log ^{2} t}\right]^{\prime}+\frac{a_{2}+\left(b_{2}+\cos _{\sigma} t\right) / \log ^{2} t}{4 t^{2}} x=0,} \\
& {\left[\frac{x^{\prime}}{a_{1}+\left(b_{1}+\cos _{\varrho} t\right) / \log ^{2} t}\right]^{\prime}+\frac{a_{2}+\left(b_{2}+\sin _{\sigma} t\right) / \log ^{2} t}{4 t^{2}} x=0,} \\
& {\left[\frac{x^{\prime}}{a_{1}+\left(b_{1}+\cos _{\varrho} t\right) / \log ^{2} t}\right]^{\prime}+\frac{a_{2}+\left(b_{2}+\cos _{\sigma} t\right) / \log ^{2} t}{4 t^{2}} x=0}
\end{aligned}
$$

are oscillatory for $a_{1} a_{2}>1$ and non-oscillatory for $a_{1} a_{2}<1$. In the limiting case $a_{1} a_{2}=1$, one can easily rewrite the considered equations in the form of (3.20), where $M(f)=b_{1} / a_{1}$ and $M(g)=a_{1} b_{2}$. Therefore, in the case $a_{1} a_{2}=1$, the above equations are oscillatory if and only if $b_{1}>a_{1}\left(1-a_{1} b_{2}\right)$.

If we know that an equation is conditionally oscillatory, then we can use it as a testing equation for many other equations. For example, using the Sturmian comparison theorem (see [14], Theorem 1.2.4), we can proceed for perturbed Euler type half-linear equations as follows. Let us consider

$$
\left[r_{0}^{-\frac{p}{q}}(t) \Phi\left(x^{\prime}\right)\right]^{\prime}+s_{0}(t) \frac{\Phi(x)}{t^{p}}+g(t) \Phi(x)=0
$$

and

$$
\left[\left[r_{0}(t)+f(t)\right]^{-\frac{p}{q}} \Phi\left(x^{\prime}\right)\right]^{\prime}+s_{0}(t) \frac{\Phi(x)}{t^{p}}=0
$$

where $f, g$ are arbitrary continuous functions and $r_{0}, s_{0}$ are $\alpha$-periodic continuous functions such that $r_{0}, f$ are positive and $M\left(r_{0}\right)=1, M\left(s_{0}\right)=q^{-p}$.

Equation (3.24) is non-oscillatory if there exist $\beta_{i}$-periodic continuous functions $S_{i}, i \in$ $\{1, \ldots, n\}$, such that

$$
M\left(\sum_{i=1}^{n} S_{i}\right)=1, \quad \sum_{i=1}^{n} S_{i}(t)>0, \quad t \in \mathbb{R},
$$

and

$$
\limsup _{t \rightarrow \infty} \frac{g(t) t^{p} \log ^{2} t}{\sum_{i=1}^{n} S_{i}(t)}<\frac{q^{1-p}}{2}
$$

Equation (3.24) is oscillatory if the functions $S_{i}$ satisfy (3.26) and

$$
\liminf _{t \rightarrow \infty} \frac{g(t) t^{p} \log ^{2} t}{\sum_{i=1}^{n} S_{i}(t)}>\frac{q^{1-p}}{2}
$$


Indeed, from inequality (3.27), we obtain $\varepsilon>0$ with the property that

$$
g(t)<\left(\frac{q^{1-p}}{2}-\varepsilon\right) \frac{\sum_{i=1}^{n} S_{i}(t)}{t^{p} \log ^{2} t}
$$

for all sufficiently large $t$. Thus, it suffices to use Theorem 3.5 and the Sturmian comparison theorem. Analogously, we get the statement concerning inequality (3.28).

Similarly, (3.25) is non-oscillatory if there exist $\alpha_{i}$-periodic continuous functions $R_{i}$ for $i \in\{1, \ldots, m\}$ such that

$$
M\left(\sum_{i=1}^{m} R_{i}\right)=1, \quad \sum_{i=1}^{m} R_{i}(t)>0, \quad t \in \mathbb{R},
$$

and we have

$$
\limsup _{t \rightarrow \infty} \frac{f(t) \log ^{2} t}{\sum_{i=1}^{m} R_{i}(t)}<\frac{q^{2}}{2 p}
$$

On the other hand, if the functions $R_{i}$ satisfy (3.29) and

$$
\liminf _{t \rightarrow \infty} \frac{f(t) \log ^{2} t}{\sum_{i=1}^{m} R_{i}(t)}>\frac{q^{2}}{2 p}
$$

then (3.25) is oscillatory.

\section{Competing interests}

The authors declare that they have no competing interests.

\section{Authors' contributions}

The authors declare that the research was realized in collaboration with the same responsibility and contributions. All authors read and approved the final manuscript.

\section{Acknowledgements}

The first author is supported by Grant P201/10/1032 of the Czech Science Foundation. The second author is supported by the project 'Employment of Best Young Scientists for International Cooperation Empowerment'

(CZ.1.07/2.3.00/30.0037) co-financed from European Social Fund and the state budget of the Czech Republic. The authors would like to thank the referees for their comments which improved the presentation of the results.

Received: 25 February 2015 Accepted: 8 June 2015 Published online: 20 June 2015

\section{References}

1. Kneser, A: Untersuchungen über die reellen Nullstellen der Integrale linearer Differentialgleichungen. Math. Ann. 42(3), 409-435 (1893). doi:10.1007/BF01444165

2. Gesztesy, F, Ünal, M: Perturbative oscillation criteria and Hardy-type inequalities. Math. Nachr. 189, 121-144 (1998). doi:10.1002/mana.19981890108

3. Schmidt, KM: Oscillation of perturbed Hill equation and lower spectrum of radially periodic Schrödinger operators in the plane. Proc. Am. Math. Soc. 127, 2367-2374 (1999). doi:10.1090/S0002-9939-99-05069-8

4. Schmidt, KM: Critical coupling constant and eigenvalue asymptotics of perturbed periodic Sturm-Liouville operators. Commun. Math. Phys. 211, 465-485 (2000). doi:10.1007/s002200050822

5. Krüger, H, Teschl, G: Effective Prüfer angles and relative oscillation criteria. J. Differ. Equ. 245(12), 3823-3848 (2008) doi:10.1016/j.jde.2008.06.004

6. Krüger, H, Teschl, G: Relative oscillation theory for Sturm-Liouville operators extended. J. Funct. Anal. 254(6) 1702-1720 (2008). doi:10.1016/j.jfa.2007.10.007

7. Krüger, H, Teschl, G: Relative oscillation theory, weighted zeros of the Wronskian, and the spectral shift function. Commun. Math. Phys. 287(2), 613-640 (2009). doi:10.1007/s00220-008-0600-8

8. Elbert, Á: Oscillation and nonoscillation theorems for some nonlinear ordinary differential equations. In: Ordinary and Partial Differential Equations, Dundee, 1982. Lecture Notes in Math., vol. 964, pp. 187-212. Springer, Berlin (1982)

9. Elbert, Á: Asymptotic behaviour of autonomous half-linear differential systems on the plane. Studia Sci. Math. Hung. $19(2-4), 447-464(1984)$ 
10. Hasil, P: Conditional oscillation of half-linear differential equations with periodic coefficients. Arch. Math. 44(2), 119-131 (2008)

11. Hasil, P, Veselý, M: Oscillation of half-linear differential equations with asymptotically almost periodic coefficients. Adv. Differ. Equ. 2013, Article ID 122 (2013). doi:10.1186/1687-1847-2013-122

12. Veselý, M, Hasil, P, Mařík, R: Conditional oscillation of half-linear differential equations with coefficients having mean values. Abstr. Appl. Anal. 2014, Article ID 258159 (2014). doi:10.1155/2014/258159

13. Agarwal, RP, Grace, SR, O'Regan, D: Oscillation Theory for Second Order Linear, Half-Linear, Superlinear and Sublinear Dynamic Equations. Kluwer Academic, Dordrecht (2002). doi:10.1007/978-94-017-2515-6

14. Došlý, O, Řehák, P: Half-Linear Differential Equations. Elsevier, Amsterdam (2005)

15. Došlý, O, Hasil, P: Critical oscillation constant for half-linear differential equations with periodic coefficients. Ann. Mat. Pura Appl. 190(3), 395-408 (2011). doi:10.1007/s10231-010-0155-0

16. Došlý, O, Funková, H: Perturbations of half-linear Euler differential equation and transformations of modified Riccati equation. Abstr. Appl. Anal. 2012, Article ID 738472 (2012). doi:10.1155/2012/738472

17. Došlý, O, Funková, H: Euler type half-linear differential equation with periodic coefficients. Abstr. Appl. Anal. 2013, Article ID 714263 (2013). doi:10.1155/2013/714263

18. Došlý, O, Veselý, M: Oscillation and non-oscillation of Euler type half-linear differential equations. J. Math. Anal. Appl. 429(1), 602-621 (2015)

19. Veselý, M, Hasil, P: Conditional oscillation of Riemann-Weber half-linear differential equations with asymptotically almost periodic coefficients. Studia Sci. Math. Hung. 51(3), 303-321 (2014). doi:10.1556/SScMath.51.2014.3.1283

20. Elbert, Á, Schneider, A: Perturbations of half-linear Euler differential equation. Results Math. 37(1-2), 56-83 (2000), doi:10.1007/BF03322512

21. Hasil, P, Veselý, M: Critical oscillation constant for difference equations with almost periodic coefficients. Abstr. Appl. Anal. 2012, Article ID 471435 (2012). doi:10.1155/2012/471435

22. Naiman, PB: The set of isolated points of increase of the spectral function pertaining to a limit-constant Jacobi matrix Izv. Vysš. Učebn. Zaved., Mat. 1959(1(8)), 129-135 (1959)

23. Veselý, M, Hasil, P: Oscillation and non-oscillation of asymptotically almost periodic half-linear difference equations. Abstr. Appl. Anal. 2013, Article ID 432936 (2013). doi:10.1155/2013/432936

24. Hasil, P, Vítovec, J: Conditional oscillation of half-linear Euler-type dynamic equations on time scales. Electron. J. Qual. Theory Differ. Equ. 2015, 6 (2015)

25. Vítovec, J: Critical oscillation constant for Euler-type dynamic equations on time scales. Appl. Math. Comput. 243(7), 838-848 (2014). doi:10.1016/j.amc.2014.06.066

26. Veselý, M, Hasil, P: Non-oscillation of half-linear differential equations with periodic coefficients. Electron. J. Qual. Theory Differ. Equ. 2015, 1 (2015)

\section{Submit your manuscript to a SpringerOpen ${ }^{\circ}$ journal and benefit from:}

- Convenient online submission

- Rigorous peer review

- Immediate publication on acceptance

- Open access: articles freely available online

- High visibility within the field

- Retaining the copyright to your article 